\title{
Introducing a clerkship curriculum based on entrustable professional activities: a pilot study
}

Severin Pinilla ( $\nabla$ severin.pinilla@upd.unibe.ch )

Institute for Medical Education https://orcid.org/0000-0002-0797-2043

Andrea Cantisani

University Hospital of Psychiatry and Psychotherapy, Bern

Stefan Klöppel

University Hospital of Old Age Psychiatry and Psychotherapy Bern

Werner Strik

University Hospital of Psychiatry and Psychotherapy Bern

Christoph Nissen

University Hospital of Psychiatry and Psychotherapy Bern

Sören Huwendiek

Institut für medizinische Lehre (IML) Bern

Research article

Keywords: clerkship, entrustable professional activities, educational outcomes

Posted Date: May 12th, 2020

DOl: https://doi.org/10.21203/rs.3.rs-25193/v1

License: (c) (i) This work is licensed under a Creative Commons Attribution 4.0 International License.

Read Full License 


\section{Abstract}

\section{Background}

General clerkship quality criteria have not been studied after introducing a curriculum based on entrustable professional activities (EPAs). Therefore, we conducted a pilot study to explore educational outcomes of an EPA-based clerkship curriculum reform.

\section{Methods}

We collected multiple self-assessment and evaluation data on levels 1-3 of the New World Kirkpatrick model (KPM). For level 1 (reaction) we analyzed curriculum evaluations and verbal and written students' feedback. For level 2 (learning) pre- and post-clerkship self-assessments of perceived need for supervision for each EPA and students' written learning reflections based on patient mix exposure were analyzed. For level 3 (behavior) we evaluated workplace-based assessments.

\section{Results}

The first student cohort in the new EPA-based clerkship completed 180 self-assessments (18 per student) of need for supervision and 63 documented workplace-based assessments (average of 6.3 per student in four weeks). On KPM level 1 we saw a high overall satisfaction with the clerkship (average of 4.9, range: 4.0-5.0 on a 5-point Likert scale). In written evaluations and feedback rounds students pointed out the importance of structured bedside teaching in the first clerkship week. On KPM level 2 the overall decrease of self-assessed need for supervision before and after the clerkship was two supervision levels (from direct to indirect supervision) and statistically significant $(p<0.05)$. For three EPAs students reached indirect supervision levels. Learning reflections and patient mix analysis indicated that students were exposed to a wide range of diagnostic categories (ICD-10: F0 - F6) and received actionable feedback for communication skills. On KPM level 3 clinical supervisors predominantly used EPAs 1 (History taking), 2 (Assessing mental status) and 8 (Documentation and presentation) for workplace-based assessments. We saw a decreasing need for supervision from the first to the last week in the clerkship according to the supervisors' judgements.

\section{Conclusion}

Students reacted positively to introducing an EPA-based clerkship curriculum. The EPA-oriented formative self-assessments and workplace-based assessments seemed to support achievement of competencybased learning goals. However, more in-depth understanding of the entrustment process in the clerkship context is necessary to fully leverage the potential of an EPA-based clerkship.

\section{Background}

A new competency-based national learning catalogue has been introduced in Switzerland for undergraduate medical education [1]. The PROFILES-catalogue (Principal Relevant Objectives and 
Framework for Integrated Learning and Education in Switzerland) includes nine entrustable professional activities (EPAs) and serves as a blueprint for clinical curricula in undergraduate medical education (UME). However, there is currently only limited evidence available on how to introduce and work with EPAs in clinical rotations, such as clerkships. While there has been attention to implementing EPA-based curricula from a general health professions and graduate medical education perspective, little attention has been paid to general quality criteria of clerkships after introducing EPAs [2, 3].

Introducing an EPA-based clinical curriculum can involve selection and development of EPAs, implementation of new teaching activities and reforming assessment instruments. Clinical educators in different specialties started to work with EPAs in UME in the context of clerkships, subinternships, electives or boot-camps [4-7]. These EPA-based curricula are mostly not embedded in larger programmatic assessment frameworks [8] but are limited to few weeks of continuous or spaced clinical exposure in one clinical specialty $[9,10]$. Only few medical schools have started to implement comprehensive assessment structures to implement EPA-based UME curricula [11].

Early research in the context of EPA-based clerkship curricula has mostly focused on three aspects: development of specialty specific EPAs [12], different implementation aspects of EPAs [13] and assessment of EPAs with supervision scales and actionable feedback [2, 14-16]. Although selfassessment of competence is not reliable [17], self-assessment of self-efficacy related constructs, such as perceived need for supervision, might be valuable in primarily formative assessment contexts. We did not find studies that explored formative assessment aspects when introducing an EPA-based clerkship curriculum. Additionally, general quality criteria of clerkships were partly not considered after EPA-based curricular reforms in clerkships. These criteria include student satisfaction, perceived and observed learning progress, patient mix exposure and behavioral change [18, 19].

Our goal for this pilot study was to better understand general quality criteria and associated educational outcomes when introducing EPAs to a clerkship curriculum. Therefore, we explored multiple data sources on the first three levels of the New World Kirkpatrick model [19] of an EPA-based clerkship curriculum reform. The results from this study inform continuing curriculum reform and might be relevant in particular for clinical educators planning to or already implementing EPA-based clinical curricula as well as educators involved in larger UME curriculum design.

\section{Methods}

\section{Development of an EPA-based clerkship curriculum}

The theoretical models that informed the implementation process included concepts of entrustment in the workplace [14, 20], programmatic assessment perspectives [21] and competency-based undergraduate learning and teaching $[1,22,23]$. The Swiss national catalogue (PROFILES) is based on nine core EPAs, which are not specialty-specific [1]. A clerkship syllabus with nested EPAs for psychiatry was developed. An open-source learning management system (ILIAS) was used to collect selfassessment and evaluation data [24]. 


\section{Setting and population}

Medical students at the University of Bern, Switzerland have to complete five mandatory core clerkships during their fourth year in a six-year medical curriculum (Bachelor and Master of Medicine). Each clerkship lasts four weeks and includes the specialties of internal medicine, gynecology, pediatrics, surgery and psychiatry. Our psychiatric teaching hospital (3'800 inpatients per year, > 10'000 outpatients per year) trains about ten students per month with each teaching ward taking on one to two medical students per month ( $\mathrm{n}$ (approximately) $=100$ per academic year). The interprofessional staff of one ward typically consists of one attending physician and two residents in addition to nurses, a psychologist, a social worker, and takes care of 20-22 hospitalized patients. We use a pass-fail-grading system for clerkship students with several mandatory formative assessments (at least four workplace-based assessments, four documented patient admissions, and one scientific paper or clinical case presentation per student).

\section{Sample}

Our sample for this explorative pilot study consisted of ten medical students with an average age of 22.5 years, nine female students and one male. Eight students originated from primarily German speaking parts of the country and two from primarily French speaking parts. Two students reported prior psychiatry experience (one through clinical skills training, one through relatives with a psychiatric diagnosis). All students and clinical teaching staff were introduced through either a seminar or written information material about the EPA-based clerkship curriculum reform.

\section{Pilot study}

We used a mixed-methods explorative study design to describe the implementation process of an EPAbased core clerkship curriculum reform in psychiatry in February 2019. We used qualitative and quantitative program evaluation data collected from medical students, each spending one month on one of ten wards in a single academic teaching hospital in Bern, Switzerland. We used a published supervision scale for each nested psychiatry clerkship EPA [25] for self-assessments of need for supervision (six levels). We assessed the educational curriculum outcomes on levels 1-3 of the New World Kirkpatrick model [19]. On level 1 (satisfaction) we analyzed students' evaluation of the clerkship curriculum and students' in-between and end-of-clerkship feedback. On level 2 (learning) we analyzed self-ratings of perceived need for supervision for each EPA of the PROFILES catalogue before and after the clerkship rotation. In order to explore learning reflections based on patient mix exposure, we collected students' documentation of patient encounters including patient age, route of admission, diagnosis and perceived learning from the admission. On level 3 (behavioral change) we analyzed all supervisor rated workplace-based assessments. Assessment and evaluation data were either collected through paperbased evaluation forms or electronically via ILIAS throughout February 2019. 
The ethics committee of the canton of Bern reviewed the research design and exempted the study from additional ethical approval. Confidentiality and anonymity with regards to electronic data was maintained throughout the study. Any names or potentially identifying information were removed before analyzing the data and quotes were all translated from (Swiss-)German to English. We used the statistical software $\mathrm{R}$ to analyze supervision scale ratings with the Wilcoxon signed-rank test.

\section{Results}

\section{New EPA-based psychiatry clerkship curriculum}

A clerkship curriculum based on the PROFILES catalogue was developed. Ten students participated in the new EPA-based psychiatry curriculum and spent four weeks (approximately 50 hours per week) on ten different wards. The core EPAs with nested psychiatry clerkship EPAs are shown in Table 1. Students were introduced to the clerkship structure in a clerkship orientation seminar at the first day of the clerkship. In addition to working on the wards and taking part in routine ward and department meetings, students participated in six one-hour didactic clerkship seminars (on psychopathology, psychiatric interventions, old-age psychiatry, psychosis, stress-related disorders, personality disorders, a scientific paper presentation exercise and a scientific journal club). Furthermore, they participated in off-site visits to specialized addiction treatment facilities, which included a patients-as-teachers seminar and the child and adolescent psychiatric clinic.

KPM Level 1 educational outcome (satisfaction): Students' evaluation of the clerkship experience

Overall satisfaction with the clerkship was rated with a 5-point Likert agreement scale to the statement "I am very satisfied with the overall clerkship experience" ( $1=$ disagree, $5=$ agree) and resulted in an overall average of 4.9 (range: 4.0-5.0). Seminars and off-site visits were rated with grades ( $1=$ bad, $5=$ excellent) and received an average grade of 3.5 (range: 2.0-5.0). Students felt well integrated in their ward teams (4.8, range: 4.0-5.0 on a 5-point Likert scale) and found the online learning platform as helpful for their learning experience (4.5, range: $4.0-5.0$ on a 5-point Likert scale). They weakly agreed on whether workplace-based assessments were helpful for their learning experience (3.6, range: 2.0-5.0 on a 5-point Likert scale). Students mentioned motivation of attending physicians and residents, as well as structure and organization of the clerkship curriculum as positive aspects in their written evaluations. They emphasized the importance of structured bed-side teaching in the first clerkship week and suggested better instruction of residents with regards to workplace-based assessments as well avoiding redundancy in some of the face-to-face seminars.

KPM Level 2 educational outcome (learning): Students' self-assessment of need for supervision regarding EPAs before and after the clerkship and learning reflections

Each student rated his or her need for supervision for nine nested psychiatry clerkship EPAs in the beginning of the clerkship (asked on first day) and the end of the clerkship (asked on last day) on a 6point Likert scale (range from 1 = "I can only observe this activity", to 2 = "I can do this only as a co- 
activity with the supervisor", 3 = "I can do this activity, if the supervisor is present", $4=$ "I can do this, if the supervisor completely repeats the activity", $5=$ "I can do this, if the supervisor repeats the important parts of the activity", 6 = "I can do this, if I can ask for help when I need it"). Changes differed across EPAs and intraindividually. The average change of supervision need for all nine EPAs was 2.9, which is equivalent to moving from observing an EPA to doing an EPA independently and having a resident check the activity. However, the change in supervision level ranged from 1 to 6 . Single EPA change (except of EPA 9) and overall change of self-assessed need for supervision was statistically significant (Wilcoxon signed rank test, $p<0.05)$. Results are shown in Figure 1.

With regards to personal learning reflections, each student documented four patient admissions including admission diagnosis and personal learning effect. The full range of psychiatric disorders was covered in the clerkship. Documented admissions included schizophrenic disorders $(n=11,28 \%)$, affective disorders $(n=10,25 \%)$, substance abuse disorders $(n=5,13 \%)$, anxiety and stress related disorders $(n=5,13 \%)$, personality disorders $(n=3,8 \%)$, organic illness $(n=1,3 \%)$, eating and impulse control disorders $(n=1$, $3 \%$ ), and two admissions did not contain a diagnosis (5\%). The vast majority of written reflections addressed various aspects of communicating with psychiatric patients (e.g. "adjust communication strategy to a patient who is logorrheic and long-winded, in such a case use short and precise questions early on" or "learned how to deal with a patient who doesn't want to answer a question and refused a physical exam, communicate acceptance"). Fewer comments addressed specific aspects of the mental status exam and planning and structuring a patient admission.

KPM Level 3 educational outcome (behavioral change): Workplace-based assessments of EPAs in the clerkship

Clinical residents supervised the students and completed 63 workplace-based assessments (average of 6.3 per student). In week one $30 \%(n=19)$ EPA assessments were documented, in week two $41 \%(n=26)$, in week three $17 \%(n=11)$, and in week four $8 \%(n=5)$, two assessments were not dated $(3 \%)$. The medical faculty provided an official 3-point-Likert scale for supervision (1 = activity possible with help from supervisor, 2 = activity possible with minimal help from supervisor, $3=$ activity possible with indirect supervision). The average EPA supervision levels as evaluated by clinical supervisors per week were: 2.5 (week 1), 2.6 (week 2), 2.5 (week 3), and 3 (week 4). Of all assessments, 38\% ( $n=24)$ addressed EPA 1, $43 \%(n=27)$ addressed EPA 2, 3\% $(n=2)$ addressed EPA 3, 1.5\% $(n=1)$ addressed EPA 5, and $14 \%(n=9)$ addressed EPA 8. EPAs 4, 6, 7 and 9 were not used for workplace-based assessments. Students only partly perceived these assessments as relevant for individual learning progress (average $=3.6, S D=0.8$ on a 5-point Likert scale).

With regards to written feedback, $97 \%$ of assessment forms contained written feedback. All feedback forms were signed off by residents, except one which was signed off by a nurse. No assessment form was signed off by attending physicians, psychologists or social workers. Written feedback was predominantly formulated as a short positive affirmation of what was done well (e.g. "structured mental status examination" or "good communication during physical examination, empathic attitude"). Only few 
written feedbacks also contained actionable areas of future improvement (e.g. "try to interrupt logorrheic patient next time" or "add disorder specific questions when interviewing relatives, such as memory function in suspected dementia"). Comparing and contrasting written feedback per student indicated that supervisors in some cases referred back to previous feedbacks and validated progress (e.g. from "practice written summary of mental status" to "precise summary of mental status, pay attention to relevant information for other team members, such as housing, financial situation and family situation).

\section{Discussion}

We used mixed-methods to explore experiences with and educational outcomes of introducing an EPAbased clerkship curriculum on New World Kirpatrick's levels 1-3 [19]. Overall, the data from this pilot study indicate positive learning outcomes on various self-reported measures, including general clerkship satisfaction and a decrease in perceived need for supervision across nine EPAs. The trend for workplacebased assessments also indicated a decrease of need for supervision. However, clinical supervisors predominantly assessed EPAs 1 (Take a patient's psychiatric history), 2 (Assess mental status) and 8 (Document and present a clinical encounter with a psychiatric patient).

KPM Level 1 (satisfaction): Students' evaluation of the EPA-based clerkship curriculum experience

The overall students' experience with an EPA-based clerkship curriculum was positive and better as compared to our overall evaluation from the previous year of the same month. Studies and educational reports from other specialties revealed similar results concerning EPAs in their clerkship curricula [15, 26, 27]. A possible explanation might be, that structuring a clerkship curriculum around EPAs provides a learning scaffold that is closer to clinical working [28].

KPM Level 2 (learning): Students' self-assessment of need for supervision regarding EPAs before and after the clerkship and learning reflections

Changes in perceived need for supervision were strongest and most homogenous for those EPAs $(1,2$ and 8 ) that had been used for workplace-based assessments. EPAs 3 (Prioritize a psychiatric differential diagnosis), 4 (Order and interpret tests for psychiatric patients), 6 (Recognize and treat psychiatric emergencies) and 7 (Prescribe and develop a management plan for a psychiatric patient) showed very heterogenous changes. EPAs 5 (Initiate involuntary treatment) and 9 (Identify and report opportunities to improve patient safety in a psychiatric hospital) showed almost no change. Potential reasons include complexity of the tasks [29] or simply curricular gaps. Further research into relevant threshold concepts to effectively support students in mastering these EPAs is needed.

To explore learning progress of clerkship students we used the patient mix, a measure typically based on patient volume and patient diversity in terms of diagnoses [18] in combination with written learning reflections. The four most frequent diagnoses that students were entrusted to admit were schizophrenic disorders, affective disorders, substance abuse disorders, and anxiety and stress related disorders. Patient mix has been shown in a systematic review to correlate positively with self-reported learning 
outcomes, such as self-confidence, comfort-level, perceived quality of the learning experience, perceived effectiveness of the rotation and instructional quality [18]. Our chosen minimal patient volume (four admissions per student) in terms of documented patient admissions and resulting learning reflections did allow for several self-reported improvements in learning outcomes.

KPM Level 3 educational outcome (behavioral change): Workplace-based assessments of EPAs in the clerkship

Workplace-based assessments were frequently documented during the clerkship and showed a slight trend towards less direct supervision for three EPAs. The other six core EPAs were not assessed during the first month of our EPA-based psychiatry clerkship. Other researchers similarly reported selective assessment of core EPAs or developing clerkship specific sets of nested EPAs [4, 30]. Probably the expectation of which EPAs should be assessed needs to be communicated more clearly to both trainees and clinical supervisors as described in the context of graduate medical education [31]. Barriers and facilitators to learning specific EPAs as well as to assess these in UME need to be better understood.

\section{Limitations}

Our pilot study is limited by the small sample and these preliminary results need to be confirmed. However, we used multiple evaluation strategies, including quantitative and qualitative data to explore early EPA-based clerkship curriculum innovations. Currently, we are collecting data for the full academic year to analyze the robustness of our findings. We already were able to use insights from this pilot study to adapt our clerkship curriculum and work with teaching residents to inform curricular changes.

Implications for practice

Several aspects from our explorative study seem relevant for clerkship directors working with EPA-based curricula. On the most basic level, our study indicates that introducing an EPA-based clerkship curriculum is feasible in smaller scale clinical teaching contexts and that students reacted positively. This might be in particular interesting for departments planning to work with EPAs before longitudinal assessment strategies for EPA-based undergraduate medical education curricula have been implemented. Selfassessment of perceived need for supervision can effectively be used in this context to explore changes for specific EPAs during a clerkship. Based on our observations the deliberate use of formative workplacebased assessments might be a potential driver of achieving learning goals with regard to EPAs.

With clinical residents as primary supervisors of clerkship students, they should receive adequate support, training and instruction for their role as clinical teachers. From a clerkship curriculum management perspective, using self-assessment of perceived need for supervision can help clerkship directors to identify weaknesses in the curriculum and adjust curriculum content accordingly. For example, we found that students seemed to struggle more with identifying differential diagnosis and formulating a management plan for psychiatric patients as compared to EPAs like taking the psychiatric history and 
assessing the mental status. Exploring underlying threshold concepts and adapting or designing new curricular elements to help students achieve these competencies are consequences of our pilot study.

\section{Conclusion}

Our pilot study indicates, that introducing an EPA-based clerkship curriculum can help to focus educational resources on competency-based learning goals. Teaching skills of clinical residents seem to be essential. Future research should further explore the entrustment process in the context of clerkships.

\section{Declarations}

\section{Ethics approval and consent to participate}

The cantonal ethics committees of Bern (Kantonale Ethikkommission Bern, Gesundheits- und Fürsorgedirektion des Kantons Bern, member of the Swiss Association of Research Ethics Committees) reviewed the research design and exempted the study from additional ethical approval (05.11.2018, project ID: 2018-01966).

\section{Consent for publication}

"Not applicable"

\section{Competing interests}

$\mathrm{SH}$ is associate editor of BMC Medical Education. All other authors declare that they have no competing interests.

\section{Funding}

“Not applicable"

\section{Authors' contributions}

SP, CN and SH conceptualized the study. SP and AC were responsible for the data collection and analysis. SK, WS and CN contributed to curriculum design, data interpretation and manuscript revision. All authors were involved in the final data synthesis and in drafting and revising the final manuscript.

\section{Acknowledgements}

We would like to thank Carmen Unglert for her exceptional support with administering and coordinating the clerkship program.

\section{Availability of data and material}

Anonymized quantitative data can be made available on request from the authors. 


\section{References}

1. Michaud, P.-A. and P. Jucker-Kupper, PROFILES; Principal Relevant Objectives and Framework for Integrated Learning and Education in Switzerland, in The Profiles Working Group. 2017, Joint Commission of the Swiss Medical Schools: Bern.

2. Shorey, S., et al., Entrustable professional activities in health care education: a scoping review. Med Educ. 2019;53:766-77.

3. O'Dowd, E., et al., A systematic review of 7 years of research on entrustable professional activities in graduate medical education, 2011-2018. Med Educ. 2019;53:234-49.

4. Klapheke, M., T. Johnson, and M. Cubero, Third-and fourth-year medical student self-assessment in entrustable professional activities. Medical Science Educator. 2017;27:159-64.

5. Vu, T.R., et al., The New Internal Medicine Subinternship Curriculum Guide: a Report from the Alliance for Academic Internal Medicine. J Gen Intern Med. 2019;34:1342-7.

6. McClintic, J.A., C.L. Snyder, and K.M. Brown, Curricular Innovation in the Surgery Clerkship: Can Assessment Methods Influence Development of Critical Thinking and Clinical Skills? J Surg Educ. 2018;75:1236-44.

7. Lamba, S., et al., A suggested emergency medicine boot camp curriculum for medical students based on the mapping of Core Entrustable Professional Activities to Emergency Medicine Level 1 milestones. Adv Med Educ Pract. 2016;7:115-24.

8. Van Der Vleuten, C.P. and L.W. Schuwirth, Assessing professional competence: from methods to programmes. Med Educ. 2005;39:309-17.

9. Hsieh, E., et al. Formative Assessment and Coaching for 3 Core EPAs in an Internal Medicine SubInternship. 2019, August. Poster session presented at the AMEE conference, Vienna, Austria.

10. Plant, R., D. Byrne, and S. Donnelly. Introduction of an Entrustable Professional Activities (EPA) framework for the Intern Year (PGY1) in Ireland: Mapping the Clinical Practice of Internship in Real Time. 2019, August. Poster session presented at the AMEE conference, Vienna, Austria.

11. Andrews, J.S.M.D., et al., Education in Pediatrics Across the Continuum (EPAC): First Steps Toward Realizing the Dream of Competency-Based Education. Acad Med. 2018;93:414-20.

12. Chen, H.C., et al., Developing entrustable professional activities for entry into clerkship. Acad Med. 2016;91:247-55.

13. Jablonover, R.S. and A. Stagnaro-Green, ECG as an entrustable professional activity: CDIM survey results, ECG teaching and assessment in the third year. The American journal of medicine. 2016;129:226-30. e1.

14. Meyer, E.G., et al., Scoping Review of Entrustable Professional Activities in Undergraduate Medical Education. Acad Med. 2019;94:1040-9.

15. Murray, K.E., et al., Crossing the gap: using competency-based assessment to determine whether learners are ready for the undergraduate-to-graduate transition. Acad Med. 2019;94:338-45. 
16. Duijn, C.C., et al., Am I ready for it? Students' perceptions of meaningful feedback on entrustable professional activities. Perspectives on medical education. 2017;6:256-64.

17. Eva, K.W. and G. Regehr, Self-assessment in the health professions: a reformulation and research agenda. Acad Med. 2005;80:S46-S54.

18. de Jong, J., et al., A systematic review of the relationship between patient mix and learning in workbased clinical settings. A BEME systematic review: BEME Guide No. 24. Med Teach. 2013;35:e1181e96.

19. Moreau, K.A., Has the new Kirkpatrick generation built a better hammer for our evaluation toolbox? Med Teach. 2017;39:999-1001.

20. ten Cate, O., et al., Curriculum development for the workplace using entrustable professional activities (EPAs): AMEE guide no. 99. Med Teach. 2015;37:983-1002.

21. van der Vleuten, C.P., et al., A model for programmatic assessment fit for purpose. Med Teach. 2012;34:205-14.

22. AAMC. Association of American Medical Colleges. Core entrustable professional activities for entering residency: Curriculum developers' guide. 2014 [cited 2019 January 1st, 2019]; Available from: https://members.aamc.org/eweb/upload/Core\%20EPA\%20Curriculum\%20Dev\%20Guide.pdf.

23. AFMC. Association of Faculties of Medicine of Canada. Entrustable professional activities for the transition from Medical School to Residency 2016 [cited 2019 January 1st, 2019]; Available from: https://afmc.ca/sites/default/files/documents/AFMC_Entrustable_Professional_Activities_EN_0.pdf.

24. Lisowska Masson, A., T. Amstutz, and D. Lalanne, A usability refactoring process for large-scale open source projects: The ILIAS case study, in Proceedings of the 2017 CHI Conference Extended Abstracts on Human Factors in Computing Systems. 2017, May: Denver, Colorado, USA. p. 1135-43.

25. Chen, C.H., S.W.E. van den Broek, and O. ten Cate, The case for use of entrustable professional activities in undergraduate medical education. Acad Med. 2015;90:431-6.

26. Schatte, D., et al., Field Guide to Boot Camp Curriculum Development. Acad Psychiatry. 2019;43:2249.

27. Jonker, G., et al., An elective entrustable professional activity-based thematic final medical school year: an appreciative inquiry study among students, graduates, and supervisors. Adv Med Educ Pract. 2018;9:837-45.

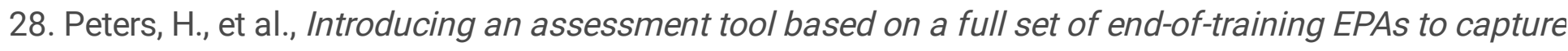
the workplace performance of final-year medical students. BMC Med Educ. 2019;19:207.

29. Khatri, R., J. Knight, and I. Wilkinson, Threshold concepts: A portal into new ways of thinking and practising in psychiatry. Med Teach. 2020;42:178-86.

30. Elnicki, D.M., et al., An entrustable professional activity (EPA)-based framework to prepare fourth-year medical students for internal medicine careers. J Gen Intern Med. 2017;32:1255-60.

31. Lörwald, A.C., et al., Influences on the implementation of Mini-CEX and DOPS for postgraduate medical trainees' learning: A grounded theory study. Med Teach. 2019;41:448-56. 


\section{Table}

Table 1 - Nested psychiatry clerkship EPAs

Entrustable Professional Psychiatry clerkship nested EPAs Activitiy (EPA) ${ }^{1}$

Average change of perceived need for supervision ${ }^{2}$

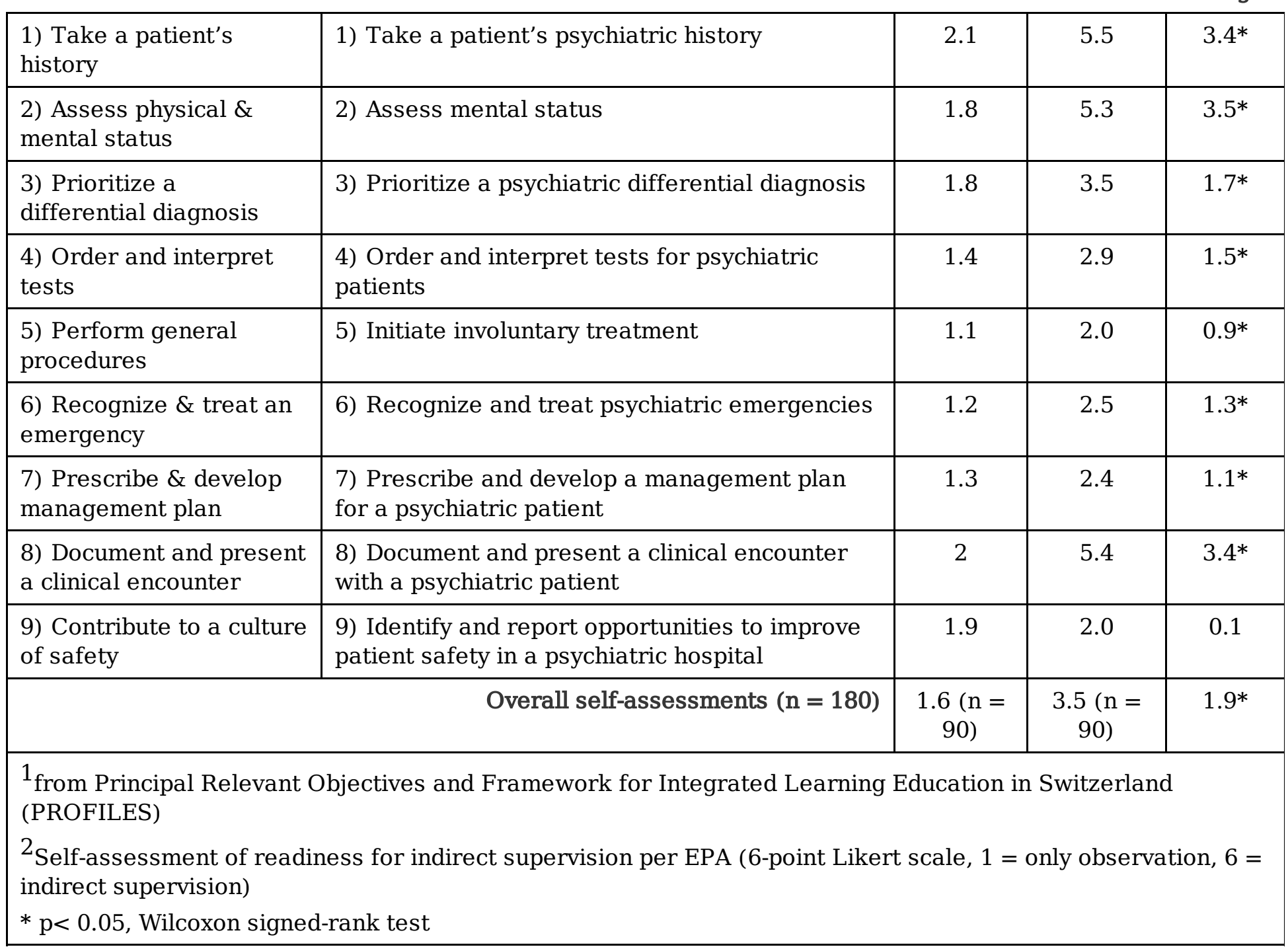

\section{Figures}


EPA 1 - Take psychiatric history EPA 2 - Assess mental status EPA 3 - Differential diagnosis
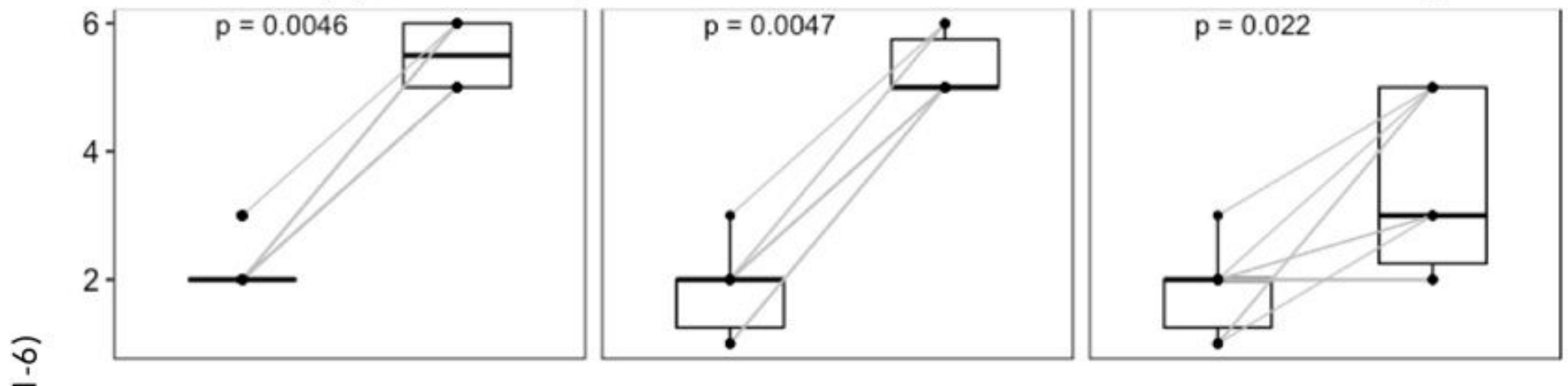

EPA 4 - Order tests

EPA 5 - Involuntary treatment

EPA 6 - Psychiatric emergencies
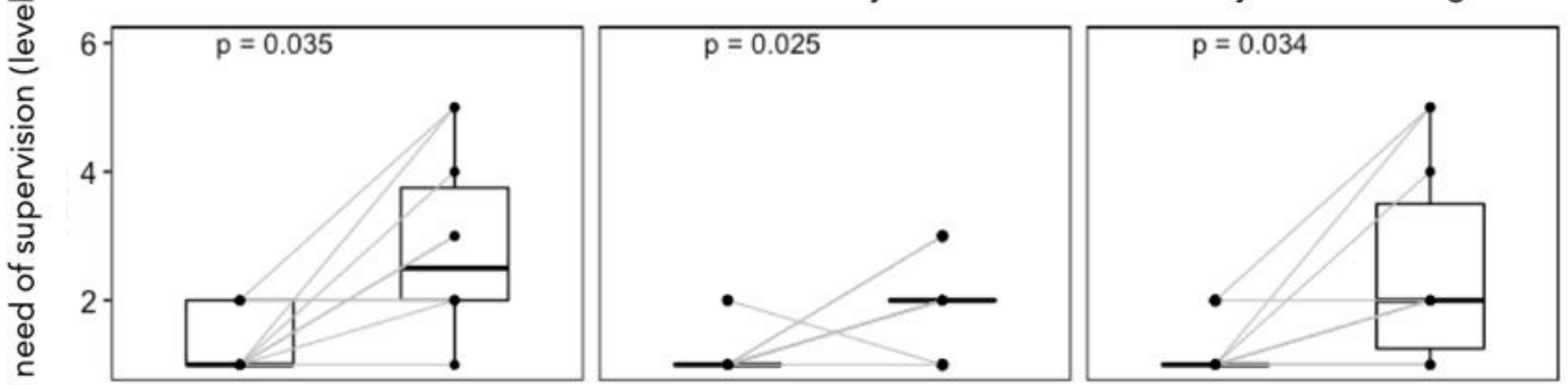

EPA 7 - Management plan

EPA 8 - Document and present EPA 9 - Safety report
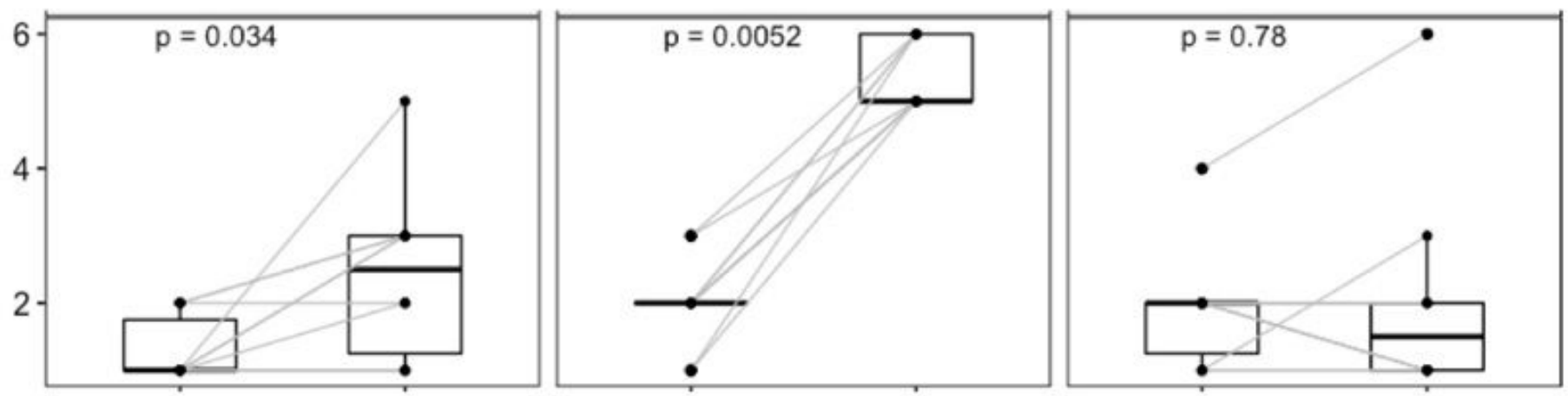

Pre-Clerkship Post-Clerkship Pre-Clerkship Post-Clerkship Pre-Clerkship Post-Clerkship

\section{Figure 1}

Changes of perceived need for supervision per EPA. Levels of supervision: 1 = "I can only observe this activity", to 2 = "I can do this only as a co-activity with the supervisor", 3 = "I can do this activity, if the supervisor is present", 4 = "I can do this, if the supervisor completely repeats the activity", 5 = "I can do this, if the supervisor repeats the important parts of the activity", 6 = "I can do this, if I can ask for help when I need it". Total of self-assessments: $n=180$ (90 pre- and 90 post-clerkship). Statistical analysis: Wilcoxon signed-rank test. 